\title{
Impact of muscle strength ladder training on disabled elders
}

\section{Ning Zhang1,2, Zheng Wang ${ }^{1,2^{*}}$, Jiaojiao Bai ${ }^{1,2^{*}}$, Ye Shen ${ }^{3}$, Yu Fu' ${ }^{4}$, and Yan Zhang ${ }^{2}$}

1. Department of Nursing, Huadong Hospital Affiliated to Fudan University, Shanghai, China

2. Clinical Key Laboratory of Geriatric Medicine, Shanghai, China

3. Health Service Center, Xianxia Community, Shanghai, China

4. Health Service Center, Bei Xinjing Community, Shanghai, China

\section{RESEARCH}

Zhang N, Wang Z, Bai J, Shen Y, Fu Y, Zhang Y. Impact of muscle strength ladder training on disabled elders. AMJ 2018;11(4):255-260.

https://doi.org/10.21767/AMJ.2018.3418

Corresponding Author:

Zheng Wang

Geriatrics Department

Huadong Hospital Affiliated to Fudan University, Shanghai, China

Tel: 86-021-18121220559

Email: zhengwang_2000@163.com

Jiaojiao Bai

Department of Nursing

Huadong Hospital Affiliated to Fudan University, Shanghai, China

Tel: 86-021-13331806987

Email: bjj163163@163.com

\section{ABSTRACT}

\section{Background}

Currently, there are many studies focusing on the physical activity program of disabled elders, but little is known about effective and cheap appliances for the disabled elderly to take home exercise.

\section{Aims}

To evaluate the impact of muscle strength ladder training on the activity ability of disabled elders.

\section{Methods}

A self-control trial was applied to 35 disabled elders in the communities in Shanghai for three months. The patients were evaluated for muscle strength and daily living activity (ADL) at the time of entry and intervention for three months.

Results

After the intervention, the patient's muscular strength grade was improved $(P<0.01)$, and the activity ability score was increased $(P<0.01)$.

\section{Conclusion}

Muscle strength ladder training could improve the muscle strength and activity ability of the upper and lower extremities. With the help of the muscle strength ladder training, the convenience, effectiveness and safety of exercise training could be increased.

\section{Key Words}

Disabled elders, strength training, training ladder, activity ability

\section{What this study adds:}

\section{What is known about this subject?}

Several exercise programs were used to improve the bad clinical outcomes of the disabled elderly, mainly including muscle strength training.

\section{What new information is offered in this study?}

This study used effective and inexpensive apparatus, muscle strength ladder for disabled elderly in muscle training at home.

3. What are the implications for research, policy, or practice?

Muscle strength ladder training would provide a simple and effective mode of exercise for community disabled elderly.

\section{Background}

Disabled elders suffer from loss of body function due to physical aging or chronic diseases, leading to inability to 
complete their daily activities independently. The life expectancy of the elderly is prolonged, the number of disabled elderly is increasing, and the demand for long-term care is increasing, which brings heavy burden to the society. With the development of China's aging process, the proportion of disabled and semi-disabled elderly in China is high and growing rapidly, which has brought serious challenges to public health. The fourth survey of the living conditions of the elderly in urban and rural areas in China ${ }^{1}$ shown that there were about 40 million 630 thousand disabled elderly in China in 2015, an increase of sevenmillion people over 2010, accounting for 18.3 per cent of the total elderly population. In terms of health care expenditure, 40 per cent medical resources were expected to be allocated to the elderly by 2050 . Disabled elders are often accompanied by a variety of adverse clinical outcomes, particularly patients with chronic diseases in bedridden elderly patients, activity and muscle strength decreased significantly ${ }^{2}$. If intervention is not applied timely, the muscle damaged degree will be aggravated, resulting in a further decline in activity. Due to this reason, disuse syndrome, respiratory tract infection, heart and lung function decline and lower limb venous thrombosis and other complications will appear. In additionally, the increased medical expenses, increased caregiver burden, will affect the quality of life of patients. ${ }^{3}$

In order to prevent and improve bad outcomes of the disabled elderly, Manini ${ }^{4}$ used the structured medium intensity activity program to intervene the patient, which included aerobic exercise, resistance exercise and flexibility training. The result has shown that the exercise plan could reduce exercise disorder. Several other studies, ${ }^{5,6}$ have shown that resistance exercise could prevent and delay the deterioration of physical function and improve the quality of life of elderly patients.

Therefore, the training of muscle strength is essential for the disabled elderly. At present, muscle strength training requires the assistance of instruments, but there is no effective cheap device for disabled elderly to do exercise at home.

\section{Aims}

According to the characteristics of disabled elderly, we used a self-developed muscle strength ladder training to stimulate the activity potential of patients, gradually helped them enhance the muscle strength of the upper and lower extremities, in order to enhance their activity ability.

\section{Methods}

\section{Trial design and participants}

The study was a self-control trial conducted in the communities in Shanghai, China, between December 2017 and February 2018. The general practitioner recruited the subjects among the contracted patients. Men and women aged $\geq 60$ years were eligible if they (1) had impaired activity, as evidenced by a ADL score 41-99; (2) had no major cognitive impairment; and (3) could safely participate in the intervention as determined by their medical history and physical examination.

A total of 35 individuals, aged $63 \sim 92$ years, with an average age of $(78.54+8.02)$ years, including 24 men (68.6 per cent), 11 women (31.4 per cent), 28 patients with stroke ( 80 per cent), and 21 patients with diabetes mellitus (21). The general situation of patients is shown in Table 1.

\section{Ethics statement}

The study protocol was approved by the ethical review committee of Huadong Hospital Affiliated to Fudan University. All participants read a statement that explained the purpose of the survey and provided written informed consent before participation in the study.

\section{Intervention}

The intervention was based on the American Sports Medicine Association "ACSM's guidelines for exercise testing and prescription". ${ }^{7}$ With the help of the muscle strength ladder training, patients could complete the training from a lying to sitting position. The device helped step up training, also helped complete the training of five to seven steps to the sitting position. Repeat five times as a set of actions. The nurse would train the patient individually once, for then, the patient would be required to have four independent exercises himself under supervision to ensure his or her safety and effectiveness. The intervention time should be at least five days a week, 15 minutes per day, twice a day, and last for three months totally. The training time should take place one hour after the meal. The general practitioner was responsible for the whole course of disease management, time arrangement of exercise training and compliance follow-up.

\section{Self-developed device}

This study adopted self-developed muscle strength ladder training, including two flexible ladders and a plurality of flexible ladder section for patients to hold. All the flexible ladder sections are distributed between two flexible ladder frames, and the ends of each flexible ladder joint are respectively connected with two flexible ladder frames. One 
end of the flexible ladder rack is provided with a connecting piece for connecting the nursing bed. After connecting one end of the new muscle training ladder to the nursing bed, the patient can pull the flexible ladder joint through the arm to change and adjust his position, which help gradually increase his own muscle strength. It comprises two flexible ladders (1) and a plurality of flexible ladder section (2) for patients (4) with tension to hold the entire flexible ladder section located between the two flexible ladders. In order to facilitate the muscle training, the ladder is fixed on the nursing bed. The connection pieces are two binding belts (3), one of which is connected with a flexible ladder rack, and the other one is connected with another flexible ladder rack.

In order to improve the comfort of the patient with a flexible ladder, a rubber hose is provided on the flexible ladder. The entire flexible ladder section is composed of a flat rope. By using this design, it will be more convenient for the medical staff or patients to collate and deposit. As for the optimization design of the rope, the rope is designed to have a coating on the surface of the silica gel layer, which can further increase the pull grip comfort, while increasing the friction pull grip. As the optimization design of rubber hose, each flexible ladder section is made of rubber materials in different colours. It is easy to help patients distinguish the flexible ladder sections, and remember which colour of the flexible ladder section for pulling grip.

Before use, check if the equipment is in good condition and if the environment for patients in supine position. Firstly, the patients take hold of a flexible ladder section to make the upper body slightly raised. Then they gradually pull grip adjacent to the flexible ladder section so that the upper body could be fully lifted, while holding a flexible ladder section. Finally, the patients move to transfer to the edge at the bedside nursing seat (6). Even though their strength is poor, they still could transfer themselves from the nursing bed to the nearby seat on their own.

Figure 1 illustrates the first use state of the strength training ladder: the patient sits on a nursing bed, his legs are straight or bent, and the flexible ladder is held by both hands or a single hand, so that the upper body reciprocates back and forth. Additionally, their arm and waist muscle strength could be improved.

Figure 2 shows second use state of the muscle strength ladder training: the patient stands on the side of the nursing bed, holding the flexible ladder section with both hands or one hand, and can rely on the pulling force of the flexible ladder frame to achieve the exercise of lower limbs and arm muscles. In addition, the patient can pull the flexible ladder step by step, so that he can walk forward or step back to exercise his own lower limb muscle strength.

\section{Measures}

Participants were evaluated at baseline and three-month clinic visits by community nurses. Home, telephone, and proxy assessments were attempted if the participant could not come to the clinic.

Activity of Daily Living Scale (ADL): The Barthel Index ${ }^{8}$ is currently the most widely used method of assessment of daily living activities in clinical practice. The scale contains a total of 10 items, basically covering all aspects of daily life in response to physical function, each activity. Ratings are graded, and different levels represent various independent ability. The higher the level, the higher the ability to represent independence. Grades of different items correspond to different scores. Among them, the highest score for grooming and bathing is five points, and the highest score is 10 points for eating, toileting, dressing, controlling bowel movements, controlling urination, and going up and down the stairs, and the highest score for bed and chair transfers is 15 Points, total score 100 points. The higher the score, the better the functional status and the less dependent. Score criteria: Total score $\leq 40$ points, heavily dependent; Total score 41-60 points, moderately dependent; Total score 61-99 points, mildly dependent; Total score 100 points, no need to rely on.

Muscle Strength Grading: Based on the muscle strength classification criteria, ${ }^{9}$ the prognoses of the patients with prosthetic muscles were graded on six levels: 0-V. Level 0: The muscles are completely paralyzed and the palpation muscles have no contractile force; Level I: The muscles have active contraction but cannot drive the joint activities; Level II: Can drive the joints horizontally, but not against the gravity; Level III: Can resist gravity and do active joint activities, but cannot resist resistance. The limbs can overcome the absorption of the earth's heart and can lift off the bed; Level IV: can resist greater resistance, but weaker than normal; V: normal muscle strength.

\section{Data analysis}

SPSS 20.0 was used for statistical analysis. Measured data were expressed as mean change \pm standard deviation. The t-test was used for comparison between groups. Count data were expressed using frequency and composition ratio, and $\chi 2$ test was used for comparison between groups. The difference was statistically significant at $P<0.05$. 


\section{Results}

There were six patients (17.1 per cent) who had intervened before grade 2 muscle strength, 27 patients ( 77.1 per cent) with grade 3 muscle strength, two patients (5.7 per cent) with grade 4 muscle strength, and 0 patients ( 0 per cent) with grade 5 muscle force. There were 0 cases ( 0 per cent) of grade 2 muscle force, seven cases (20 per cent) of grade 3 muscle strength, 26 cases ( 74.3 per cent) of grade 4 muscle strength, and two cases (5.7 per cent) of grade 5 muscle strength. Before and after the intervention, the patient's muscle strength was statistically different. The ADL evaluation score of patients before intervention (41.57 \pm 1.21$)$ points, and the average ADL scores of patients after intervention (57.29 \pm 2.05$)$ were statistically significant before and after intervention (Table 2).

\section{Discussion}

Disabled elders are those who lose their ability to take care of themselves in daily life. The level of disabled elders in China ${ }^{10}$ showed that 26.6 per cent of patients could not take a bath, 15.0 per cent of patients could not use the toilet, and 13.1 per cent of patients could not walk indoors. As a result of the weakening of mobility, there is a generalization of chronic diseases in older people who are disabled. These elderly patients need long-term care of the family and society. They gradually increase family and social burdens, and medical costs. The study has shown ${ }^{11}$ that disabled seniors could effectively improve their physical function through proper exercise training. In this trial, the disability evaluation was performed using the activity of daily living activity scale, and the self-made muscle strength ladder training was used to intervene. The elderly patients with impaired ability experienced an increase in Barthel's index with three months intervention.

Muscle strength refers to the force of contraction of muscles during active exercise. It is a guarantee for maintaining the activities of daily life of the human body. It is of great significance for maintaining the activities of the elderly in daily life and the gait stability of the lower limbs. The resistance training for the elderly can reduce muscle decay, increase muscle strength, and improve mobility. ${ }^{12}$ In this study, the self-developed muscle strength ladder training was used to perform resistance exercises, which helps patients to change their postures autonomously. The patient sat on a nursing bed and pulled the flexible ladder section to reciprocate the upper body to exercise the upper limbs and abdominal muscles. For patients who were able to get out of bed, they could be instructed to stand by the nursing bed. Afterwards, they grasped the ladder section, relied on the tension of the flexible ladder frame, could move to the seat, and could also exercise the lower limb muscle strength. From the muscle strength test results, the muscle strength of disabled patients was effectively improved.

Mild and moderate disabled elderly are impaired in their mobility and are accompanied by many chronic diseases. Resistance training has certain challenges, and individualized, safe and effective appropriate exercise is needed. The muscle strength ladder is simple and cheap, and the practice method is easy to be mastered by the patient. According to the strength of their own activities, patients can adjust themselves to the best position according to the training ladder, so as to meet the needs of personalized rehabilitation training. Each section of the ladder with different bright colours, stimulate the patient's visual senses. The disabled elderly could achieve rehabilitation step by step, according to their own conditions. At the same time, the patient can choose different ladder sections according to their own tolerance, and gradually increase the difficulty in order to achieve the standard of rehabilitation training. Although the effect was significant, due to the use of self-control design, there may be bias risk.

During the training, we had care to ensure the safety of the disabled elderly. During this study, patients had no adverse events, such as fall and skeletal muscle injury.

\section{Conclusion}

The results of the trial suggested that muscle strength ladder training could improve the muscle strength and activity ability. The device reflected the good convenience, effectiveness and safety of rehabilitation for disabled elders. It is suitable for popularization and application in more people and institutions.

\section{References}

1. Chen N, Yuan N. The social work intervention of the disabled elderly in the elderly under the threshold of energy growth is discussed. Chinese J Gerontology. 2018;(02):482-485.

2. Kilavuz A, Meseri $R$, Savas $S$, et al. Association of sarcopenia with depressive symptoms and functional status among ambulatory community-dwelling elderly. Arch Gerontol Geriatr. 2018;76:196-201.

3. Al-Butmeh S, Al-Khataib N. Mental health and quality of life of elderly people in the Bethlehem district: a crosssectional study. Lancet. 2018;391(Suppl 2):S46.

4. Manini TM, Beavers DP, Pahor M, et al. Effect of physical activity on self-reported disability in older adults: Results from the Life study. J Am Geriatr Soc. 2017;65(5):980- 
988.

5. Mickle KJ, Caputi P, Potter JM, et al. Efficacy of a progressive resistance exercise program to increase toe flexor strength in older people. Clin Biomech (Bristol, Avon). 2016;40:14-19.

6. Nicholson VP, Mckean MR, Burkett BJ. Low-load highrepetition resistance training improves strength and gait speed in middle-aged and older adults. J Sci Med Sport. 2015;18(5):596-600.

7. Medicine ACOS, Whaley MH, Brubaker PH, et al. ACSM's guidelines for exercise testing and prescription. Physiotherapy. 2013;77(77):311.

8. Mahoney FJ, Barthel DW. Functional evaluation: The Barthel Index. Md Med J. 1965;14:61.

9. Goodpaster BH, Park SW, Harris TB, et al. The loss of skeletal muscle strength, mass, and quality in older adults: The health, aging and body composition study. J Gerontol A Biol Sci Med Sci. 2006;61(10):1059-1064.

10. Jing Y J, Li Y. The analysis of Chinese disabled elderly and long-term care needs. Population J. 2014;36(2):55-63.

11. Rugbeer N, Ramklass S, Mckune A, et al. The effect of group exercise frequency on health related quality of life in institutionalized elderly. Pan Afr Med J. 2017;26:35.

12. Mejíaspeña $Y$, Estébanez $B$, Rodriguezmiguelez $P$, et al. Impact of resistance training on the autophagyinflammation-apoptosis crosstalk in elderly subjects. Aging. 2017;9(2):408-418.

\section{ACKNOWLEDGEMENTS}

We appreciate that the efforts the staff and volunteers in the communities in Shanghai, China.

\section{PEER REVIEW}

Not commissioned. Externally peer reviewed.

\section{CONFLICTS OF INTEREST}

The authors declare that they have no competing interests.

\section{FUNDING}

This project was funded by Clinical Research of SHDC (NO.16CR3055A), plan of clinical skills and clinical innovation ability of municipal hospitals "Study on the application and extension of isometric resistance muscle strength training for the prevention and treatment of the elderly sarcopenia.", Shanghai Municipal Commission of Health and Family Planning (NO.20154Y0211), Scientific research subject "Study on the screening and related risk factors of the elderly sarcopenia." and the Key Project of Fudan-Fuxin Nursing Research Fund (NO. FNF201722).

\section{ETHICS COMMITTEE APPROVAL}

The study protocol was approved by Ethical Review Committee of Huadong Hospital Affiliated to Fudan University (NO.2017K017).

\section{AUTHOR CONTRIBUTIONS}

Bai J participated in the design of the study, particularly in the definition of rehabilitative interventions and clinical outcome measures. Wang Z participated in the design of the device. Shen $Y$ and $F u Y$ defined the procedure for assessment. Zhang Y planned the statistical analysis. Zhang $\mathrm{N}$ drafted the manuscript. All authors read and approved the final manuscript. 
Figure 1: Climbing staircase training in bed

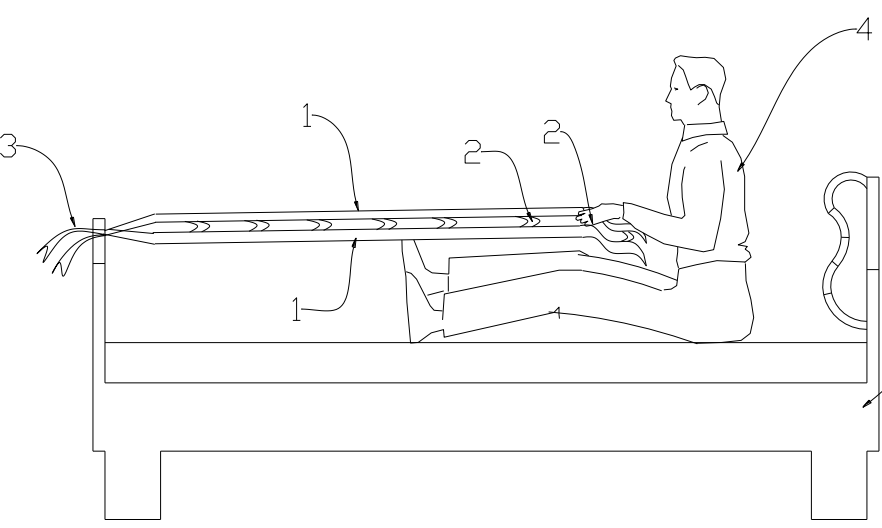

* 1- Flexible staircase; 2- Flexible Ladder; 3- Binding band; 4- Patients; 5- Nursing Bed

Figure 2: Bedside climbing staircase training

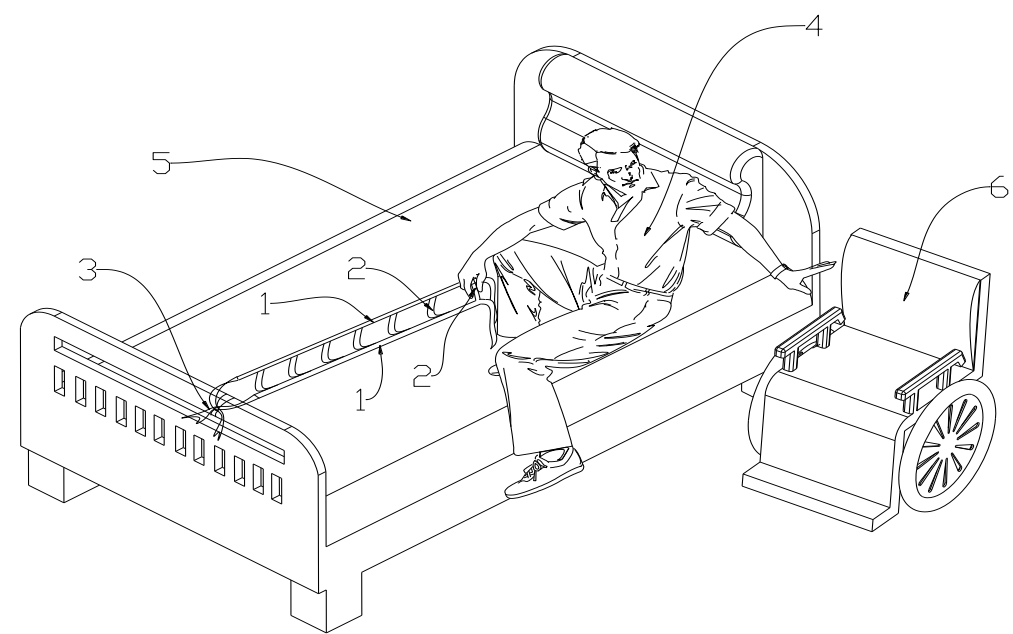

*1- Flexible staircase; 2- Flexible ladder; 3- Binding band; 4- Patients; 5- Nursing bed; 6- Wheelchair

Table 1: General condition of patients

\begin{tabular}{|c|c|c|c|c|c|c|}
\hline \multicolumn{2}{|c|}{$\operatorname{Sex}(n / \%)$} & \multicolumn{2}{|c|}{ Stroke (n/\%) } & \multicolumn{2}{|c|}{ Diabetes (n/\%) } & \multirow[t]{2}{*}{ Age $(\bar{X} \pm S)$} \\
\hline Male & Female & $Y$ & $\mathrm{~N}$ & $Y$ & $\mathrm{~N}$ & \\
\hline $24 / 68.6$ & $11 / 31.4$ & $28 / 80$ & $7 / 20$ & $21 / 60$ & $14 / 40$ & $78.54 \pm 8.02$ \\
\hline
\end{tabular}

Table 2: Changes of muscle strength and ADL

\begin{tabular}{|c|c|c|c|c|c|}
\hline & \multicolumn{4}{|c|}{ Muscular strength (n/\%) } & \multirow[t]{2}{*}{$A D L(\bar{X} \pm S)$} \\
\hline & 2 level & 3 level & 4 level & 5 level & \\
\hline 0 month & $6 / 17.1$ & $27 / 77.1$ & $2 / 5.7$ & $0 / 0$ & $41.57 \pm 1.21$ \\
\hline 3 months & $0 / 0$ & $7 / 20$ & $26 / 74.3$ & $2 / 5.7$ & $57.29 \pm 2.05$ \\
\hline $\mathrm{x}^{2} / \mathrm{t}$ & \multicolumn{4}{|c|}{40.34} & -6.60 \\
\hline$P$ & \multicolumn{4}{|c|}{$<0.01$} & $<0.01$ \\
\hline
\end{tabular}

